\title{
Efeitos da nutrição parenteral ou enteral, associadas ou não à glutamina, sobre a motilidade gastrintestinal em equinos submetidos à inanição e realimentação*,**
}

\section{Effects of parenteral or enteral nutrition, associated or not with glutamine, on gastrointestinal motility in horses submmited to starvation and refeeding}

\author{
Ubiratan Pereira de Melo, ${ }^{* * *}$ Maristela Silveira Palhares, ${ }^{* * *}$ Cíntia Ferreira, ${ }^{* * *}$ Valentim Arabicano Gheller, ${ }^{* * * *}$ \\ Fabíola Oliveira Paes Leme ${ }^{\star * * *}$
}

\begin{abstract}
Resumo
Este estudo teve por objetivo avaliar os efeitos da nutrição parenteral total ou enteral, associadas ou não à glutamina, sobre a motilidade gastrintestinal em equinos submetidos à inanição e realimentação. Foram utilizados 16 equinos adultos hígidos, sem raça definida, de ambos os sexos, quatro machos e 12 fêmeas, com idade variando entre quatro e 14 anos e peso corporal médio de 248,40 $\pm 2,28 \mathrm{~kg}$, divididos em quatro grupos, quatro animais por grupo: Grupo I (ENTGL): fluidoterapia enteral com eletrólitos associada a glutamina; Grupo II (PARGL): Nutrição parenteral total (NPT) associada a glutamina; Grupo III (ENTFL): fluidoterapia enteral com eletrólitos; Grupo IV (PARFL): fluidoterapia parenteral. O delineamento experimental foi inteiramente ao acaso, em um esquema fatorial 4x12 (grupos x tempo de colheita), para cada fase, e suas médias comparadas pelo teste de Duncan ao nível de $5 \%$ de significância. Independente do grupo experimental ocorreu redução da motilidade gastrintestinal durante a fase de inanição, mais pronunciada nos grupos PARGL e PARFL. Uma vez restabelecida a alimentação a motilidade gastrintestinal retornou à normalidade.
\end{abstract}

Palavras-Chave: equino, glicose, alimentação, suporte nutricional, trânsito intestinal.

\begin{abstract}
This study aimed to evaluate the effects of enteral or total parenteral nutrition, associated or not with glutamine, on gastrointestinal motility in horses subjected to starvation and refeeding. 16 healthy, mixed-breed adult horses of both sexes, four geldings and 12 mares, with ages ranging from four to 14 years and an average body weight of $248.40 \pm 2.28 \mathrm{~kg}$, were divided into four groups, four animals per group: Group I (ENTGL): enteral fluid therapy with electrolytes associated with glutamine; Group /I (PARGL): total parenteral nutrition (TPN) associated with glutamine; Group III (ENTFL): enteral fluid therapy with electrolytes; Group IV (PARFL): parenteral fluid therapy. The experimental design was entirely randomized, in a $4 \times 12$ factorial scheme (groups $\mathrm{x}$ harvest time), for each phase, and their means compared by the Duncan test at the level of $5 \%$ significance. Regardless of the experimental group, there was a reduction in gastrointestinal motility during the starvation phase, which was more pronounced in the PARGL and PARFL groups. Once the food was restored, gastrointestinal motility returned to normal.
\end{abstract}

Keywords: equine, glucose, feeding, nutritional support, intestinal transit.

\section{Introdução}

Vários termos têm sido utilizados na literatura visando definir situações em que os animais não ingerem alimentos (inanição, jejum, desnutrição etc.), cabendo diferenciação entre eles. Inanição (do inglês starvation) refere-se à condição na qual um animal é incapaz de ingerir alimento em decorrência de limitação extrínseca da sua disponibilidade. Já o termo jejum (do inglês fasting) refere-se à condição na qual, em decorrência de fatores intrínsecos, o animal não ingere alimento. Apesar da disponibilidade de alimentos, o animal utiliza seu tempo e energia em outras atividades como, por exemplo, termorregulação ou comportamentos relacionados à reprodução (Melo et al., 2011).

$\mathrm{Na}$ espécie equina um período variável de inanição é recomendado nos casos de doença abdominal (Melo et al., 2008; Carr, 2018), bem como antes e após procedimentos cirúrgicos para prevenir complicações à exemplo de cólica pós-operatória (Hospes \& Bleul, 2007, Bailey et al., 2016). No entanto, a retirada de alimento não é fisiológica considerandose o padrão de ingestão alimentar do equino. Sob condições

\footnotetext{
*Recebido em 11 de abril de 2021 e aceito em 22 de maio de 2021.

**Parte da tese apresentada pelo primeiro autor ao curso de Pós-graduação em Ciência Animal da Escola de Veterinária da UFMG para obtenção do título de Doutor.

***Faculdade Maurício de Nassau (Uninassau), Campus Natal/RN, Natal, Rio Grande do Norte. Autor para correspondência: ubiratan_melo@yahoo.com.br ****Escola de Veterinária da UFMG, Departamento de Clínica e Cirurgia Veterinária, Belo Horizonte, Minas Gerais.
} 
naturais, a ingestão alimentar diária a pasto dura 12 a 18 horas (Williams et al., 2015). Quando alimentado ad libitum, o tempo de jejum voluntário não excede três horas (Melo et al., 2011).

Perda progressiva de peso, fraqueza, anemia e predisposição a infecções são manifestações clínicas comuns ao estado de inanição prolongada. No aparelho digestivo são relatadas redução da produção de enzimas digestivas, atrofia da mucosa intestinal, alteração do transporte de aminoácidos através da membrana celular e redução da absorção de glutamina e arginina, o que compromete a digestão e absorção de nutrientes na fase de realimentação (Pucci et al., 2008; Lakananurak \& Gramlich, 2020).

A auscultação dos sons abdominais é parte da rotina do exame físico geral em equinos, sendo importante no estabelecimento do diagnóstico e prognóstico em casos de cólica (Salciccia et a., 2019; VanderBroek et al., 2019). A motilidade intestinal normal é controlada por fatores mioelétricos, neurais e humorais (Tapio et al., 2019). Os fatores extrínsecos e intrínsecos do sistema de controle neural são interconectados e, por sua vez, controlados por hormônios, distensão intestinal, ou estado de alimentação (Naylor et al., 2006).

O efeito do jejum prolongado ou inanição sobre os sons abdominais auscultáveis é pobremente relatado na literatura equina (Merrit et al., 1995; Naylor et al, 2006). Este assunto é particularmente importante porque a diminuição da ingestão alimentar é comum em animais doentes. Este estudo teve por objetivo avaliar os efeitos da nutrição parenteral total ou enteral, associadas ou não à glutamina, sobre a motilidade gastrintestinal em equinos submetidos à inanição e realimentação.

\section{Material e métodos}

O experimento foi realizado nas dependências da Clínica Médica de Equinos da Escola de Veterinária da Universidade Federal de Minas Gerais, localizada no município de Belo Horizonte. Este experimento foi aprovado pelo Comitê de Ética em Experimentação Animal (CETEA/UFMG) sob o número 34/2008.

Foram utilizados 16 equinos adultos hígidos, sem raça definida, de ambos os sexos, quatro machos e 12 fêmeas, com idade variando entre quatro e 14 anos e peso corporal médio de $248,40 \pm 2,28 \mathrm{~kg}$, divididos em quatro grupos, quatro animais por grupo. Para triagem e seleção dos animais foi realizado exame clínico completo conforme descrito por Speirs (1999) e exames laboratoriais (hemograma, parasitológico de fezes). Os animais passaram por tratamento endo ${ }^{1}$ e ectoparasiticida ${ }^{2}$, foram alojados em piquetes e alimentados diariamente com ração comercial ${ }^{3}(1 \mathrm{~kg} / 100 \mathrm{~kg}$ peso vivo), feno de tifton $(1 \mathrm{~kg} / 100$ kg peso vivo), além de capim elefante (Pennisetum purpureum) picado, água e sal mineral ${ }^{4}$ ad libitum. Após período de adaptação de 30 dias, os animais foram divididos aleatoriamente em quatro grupos experimentais:

Grupo I (ENTGL): fluidoterapia enteral com eletrólitos $(5,7 \mathrm{~g}$ $\mathrm{NaCl} ; 3,78 \mathrm{~g} \mathrm{NaHCO} ; 0,37 \mathrm{~g} \mathrm{KCl}$ e $10 \mathrm{~g}$ de glicose por litro de

\footnotetext{
${ }^{1}$ Ivermectina gel composto, Ouro Fino Saúde Animal, Cravinhos/SP, Brasil

${ }^{2}$ Butoxp- Intervet, Brasil

${ }^{3}$ Equitage 15P, Guabi Nutrição Animal, Brasil

${ }^{4}$ Suplemento Itambé Master Equinos, Itambé, Contagem/MG, Brasil
}

água) associada a glutamina ${ }^{5}$. Para o cálculo do volume total de fluido a ser administrado no período de 24 horas adotou-se o valor de $60 \mathrm{ml} / \mathrm{kg}$ peso vivo como taxa de manutenção. O volume total de fluido calculado foi dividido por 12 e administrado, por fluxo de gravidade, a cada duas horas via sonda nasogástrica ${ }^{6}$ calibre 11x16mm. Para o cálculo da dose total diária de glutamina a ser administrada adotou-se o valor de $0,5 \mathrm{~g} / \mathrm{kg}$ peso vivo. A quantidade total calculada de glutamina foi dividida por $12 \mathrm{e}$ administrada a cada duas horas diluída na fluidoterapia enteral. $O$ volume de fluido a ser administrado diariamente, bem como a quantidade de glutamina foram corrigidas diariamente de acordo com o peso do animal;

Grupo II (PARGL): Nutrição parenteral total (NPT) associada a glutamina. A nutrição parenteral total foi preparada a partir de soluções de aminoácidos ${ }^{7}$, lipídeos ${ }^{8}$ e glicose ${ }^{9} 50 \%$ em iguais proporções (33,33\% aminoácidos, 33,33\% de lipídeos e 33,33\% de glicose $50 \%$ ) de modo a fornecer a necessidade energética de manutenção diária.

A glutamina foi administrada na dose de $0,5 \mathrm{~g} / \mathrm{kg}$ de peso vivo por via intravenosa como uma solução a $1,5 \%$ estéril $(15 \mathrm{~g} / \mathrm{l})$. A quantidade total da NPT, bem como a quantidade de glutamina a ser administrada foram corrigidas diariamente de acordo com o peso corporal do animal. Ambas as soluções foram administradas em infusão contínua via acesso venoso na veia jugular externa direita. A velocidade de administração $(\mathrm{ml} / \mathrm{h})$ foi calculada, dividindo-se o volume total a ser infundido por 24. Fluidoterapia intravenosa utilizando solução ringer com lactato foi instituída em quantidade necessária para completar a necessidade de manutenção de $60 \mathrm{ml} / \mathrm{kg}$.

Grupo III (ENTFL): Fluidoterapia enteral com eletrólitos (5,7g $\mathrm{NaCl} ; 3,78 \mathrm{~g} \mathrm{NaHCO} ; 0,37 \mathrm{~g} \mathrm{KCl}$ e $10 \mathrm{~g}$ de glicose por litro de água). Para o cálculo do volume total de fluido a ser administrado no período de 24 horas adotou-se o valor de $60 \mathrm{ml} / \mathrm{kg}$ como taxa de manutenção. $\mathrm{O}$ volume total de fluido calculado foi dividido por 12 e administrado, por fluxo de gravidade, a cada duas horas via sonda nasogástrica calibre $11 \times 16 \mathrm{~mm}$. O volume de fluido a ser administrado foi corrigido diariamente de acordo com o peso do animal.

Grupo IV (PARFL): Fluidoterapia parenteral. O volume de fluido a ser administrado foi de $60 \mathrm{ml} / \mathrm{kg}$ de peso vivo, sendo metade do volume fornecido pela solução ringer com lactato ${ }^{10}$ e metade pela solução fisiológica ${ }^{11}$ de cloreto de sódio $0,9 \%$. Glicose $50 \%$ foi fornecida na dose de $1,5 \mathrm{~g} / \mathrm{kg}$ de peso vivo diluída na solução fisiológica. Ambas as soluções foram administradas em infusão contínua via acesso venoso na veia jugular externa direita.

O acesso venoso para infusão da NPT (grupo PARGL) e fluidoterapia parenteral (grupo PARFL) foi obtido por meio da utilização de cateter de duplo lúmen ${ }^{12}$ coaxial tamanho 12 gauge e $20 \mathrm{~cm}$ de comprimento.

${ }^{5}$ L-Glutamina, Ajinomoto do Brasil Indústria e Comércio de Alimentos Ltda, Laranjal Paulista/SP, Brasil

${ }^{6}$ Sonda nasogástrica para equinos, Provar Produtos Veterinários, Brasil

${ }^{7}$ Aminoven $10 \%$, Fresenius Kabi, Barueri/SP, Brasil

${ }^{8}$ Lipovenos 20\%, Fresenius Kabi, Barueri/SP, Brasil

${ }^{9}$ Glicose $50 \%$, Fresenius Kabi, Barueri/SP, Brasil

${ }^{10}$ Lactato de Ringer Fresenius, Fresenius Kabi, Barueri/SP, Brasil

${ }^{11}$ Solução salina fisiológica 0,9\%, Fresenius Kabi, Barueri/SP, Brasil

${ }^{12}$ Duocath - 12F, Intra Special Catheters, Germany 
Amostras intestinais foram colhidas para posterior avaliação dos efeitos da inanição e tipo de suporte nutricional sobre as características ultra-estruturais da mucosa do intestino delgado e cólon maior por meio de duas laparotomias pelo flanco, uma no início do período experimental e outra no final da fase de inanição, com intervalo de seis dias (dados não publicados).

Este estudo foi dividido em duas fases: fase $1 \mathrm{e}$ fase 2. Durante a fase 1 os animais não receberam alimento ou água além do descrito no grupo. A fase 2 consistiu na realimentação dos animais. Decorridas 12 horas da segunda laparotomia, a alimentação foi gradualmente reintroduzida. No primeiro dia foram fornecidos $4 \mathrm{~kg}$ de feno de tifton, divididos em duas refeições de $2 \mathrm{~kg}$ fornecida com 12 horas de intervalo, e $0,5 \mathrm{~kg}$ de concentrado. No segundo dia, a quantidade de feno foi aumentada para 8 quilos de feno, fornecidos em duas refeições de $4 \mathrm{~kg}$ cada, além de $1 \mathrm{~kg}$ de concentrado comercial. A partir do terceiro dia, as quantidades de alimento fornecidas foram iguais a do período de adaptação. Cada fase teve uma duração total de 144 horas (seis dias).

A motilidade gastrintestinal foi avaliada por meio da auscultação abdominal, na qual procedeu-se a contagem do número de movimentos propulsivos por minuto nos quadrantes superior e inferior direito, e superior e inferior esquerdo. Os parâmetros foram avaliados a cada 12 horas durante todo o período experimental e anotados em fichas individuais, totalizando 24 avaliações. O delineamento experimental foi inteiramente ao acaso, em um esquema fatorial $4 \times 12$ (grupos $\mathrm{x}$ tempo de colheita), para cada fase. Os dados foram tabulados em planilha $\operatorname{Exce}^{\circledR}$ e após teste de normalidade foram submetidos à análise de variância (Proc GLM) e suas médias comparadas pelo teste de Duncan ao nível de $5 \%$ de significância.

\section{Resultados e discussão}

Não foi identificada interação grupo e tempo $(p>0,05)$ em quaisquer dos quadrantes abdominais. No quadrante superior direito (Figura 1) observou-se diferença $(p<0,05)$ entre o total dos grupos na fase $1 \mathrm{e} o$ total dos tempos em ambas as fases. $O$ total dos grupos não apresentou diferença $(p>0,05)$ na fase 2. Já no quadrante inferior direito (Figura 2) observaram-se diferenças $(p<0,05)$ tanto entre o total dos grupos quanto entre $o$ total dos tempos em ambas as fases, inanição e realimentação.

Nos dois quadrantes foi observada diminuição numérica dos movimentos gastrintestinais propulsivos ao longo da fase 1 , seguida da elevação do número de movimentos gastrintestinais na fase de realimentação. Apesar de não ter sido identificada interação entre grupos e tempos, observou-se clinicamente que a diminuição dos movimentos gastrintestinais ocorreu de forma precoce nos grupos PARFL e PARGL. Essa observação clínica pode estar relacionada com a manutenção da ativação do reflexo gastro-cólico após a administração das soluções enterais nos grupos ENTFL e ENTGL. No entanto, a administração periódica da fluidoterapia enteral, independente do grupo, não impediu o desenvolvimento de hipomotilidade intestinal.

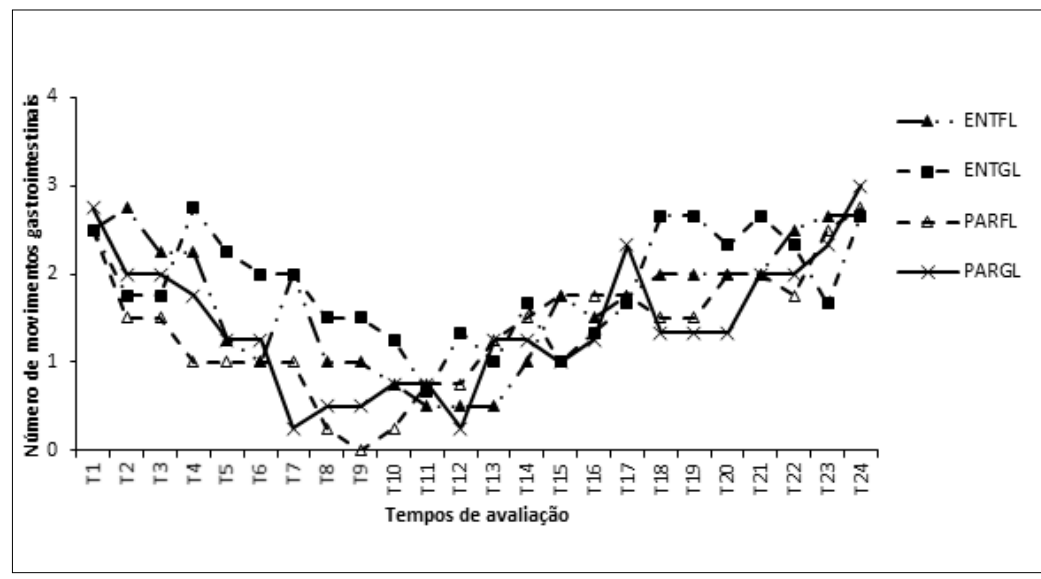

Figura 1: Número de movimentos gastrintestinais propulsivos por minuto no quadrante super direito de equinos sob inanição submetidos à fluidoterapia parenteral (PARFL)

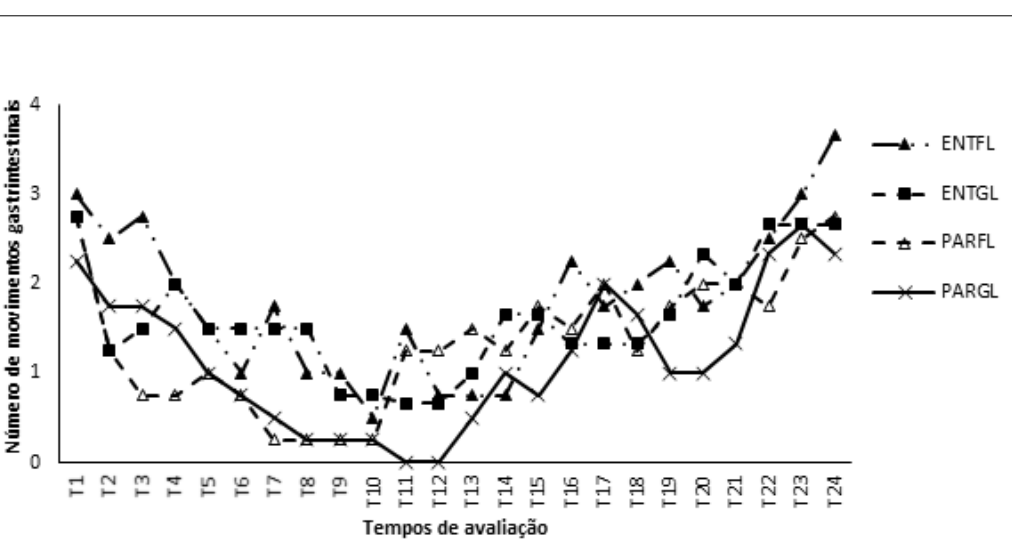

Figura 2: Número de movimentos gastrintestinais propulsivos por minuto no quadrante inferior direito de equinos sob inanição submetidos à fluidoterapia parenteral (PARFL) tal associada à glutamina (PARGL).

A auscultação do quadrante abdominal superior direito permite a auscultação do movimento de passagem da ingesta e líquidos do intestino delgado para o ceco, o que resulta na auscultação dos movimentos gastrintestinais assemelhando-se a sons de descarga. A diminuição gradativa dos sons gastrintestinais neste segmento pode representar tanto a presença de inflamação do intestino delgado resultando em quadro de íleo quanto a ausência da ingestão alimentar ou hídrica (Melo et al., 2007; Lisowski et al., 2018).

Apesar do íleo adinâmico, principalmente do intestino delgado, ser complicação comum no período pós-operatório na espécie equina (Melo et al., 2007), não foi observado quadro de íleo durante a realização deste estudo, mesmo após a segunda manipulação cirúrgica para coleta das biópsias intestinais. Portanto, a diminuição dos sons gastrintestinais neste quadrante está relacionada à ausência de ingestão alimentar e vão de encontro à observação clínica de que longos períodos sem ingestão de alimentos resultam em hipomotilidade intestinal, conforme sugerido por Naylor et al. (2006).

Em relação aos quadrantes abdominais superior e inferior esquerdo, não foi identificada interação grupo e tempo $(p>0,05)$ em ambos os quadrantes. 
No quadrante superior esquerdo (Figura 3), observou-se diferença $(p<0,05)$ tanto entre o total dos grupos quanto entre o total dos tempos em ambas as fases. Já com relação ao quadrante inferior esquerdo (Figura 4), observou-se diferença $(p<0,05)$ entre o total dos grupos na fase 1 , e entre o total dos tempos em ambas as fases. Não foi identificada diferença $(p>0,05)$ entre o total dos grupos na fase 2 .
O baixo número de movimentos propulsivos no quadrante superior esquerdo, fase 1 , foi caracterizado pela atonia observada em vários animais (9/16). Apesar da controvérsia existente na literatura em relação à estrutura representativa deste foco de auscultação (Naylor et al., 2006), os sons gastrintestinais auscultados representam o trânsito da ingesta pelo cólon menor. Este segmento gastrintestinal é responsável pela formação das síbalas e absorção final de água e nutrientes na ingesta. Em associação com o quadrante superior direito, foi neste quadrante onde observou-se diminuição precoce dos movimentos gastrintestinais em comparação com os quadrantes inferiores, corroborando com os resultados de Naylor et al. (2006).

A literatura tem associado quadros de diminuição da motilidade gastrintestinal à diminuição da concentração sérica de potássio, cálcio e magnésio (Melo et al., 2007), íons importantes para a contração muscular. Ocorreu diminuição da concentração sérica tanto do potássio quanto do magnésio em todos os grupos (dados não publicados), o que poderia explicar os achados relacionados à diminuição da motilidade gastrintestinal. No entanto, os dois achados discutidos, diminuição da motilidade gastrintestinal e diminuição da concentração de potássio e magnésio estão diretamente relacionados a ausência de ingestão alimentar (Seahorn e Seahorn, 2003).

Em ambos os quadrantes, embora as diferenças só tenham sido identificadas nas médias marginais em decorrência do baixo número de animais utilizados por grupo e ao alto coeficiente de variação da variável, observou-se diminuição numérica da quantidade total de movimentos gastrintestinais propulsivos na fase da inanição seguida da elevação, também numérica, do número desses movimentos na fase de realimentação. Da mesma forma como ocorrido nos quadrantes superior e inferior direito, clinicamente observou-se diminuição precoce do número de movimentos gastrintestinais nos grupos PARFL e PARGL.

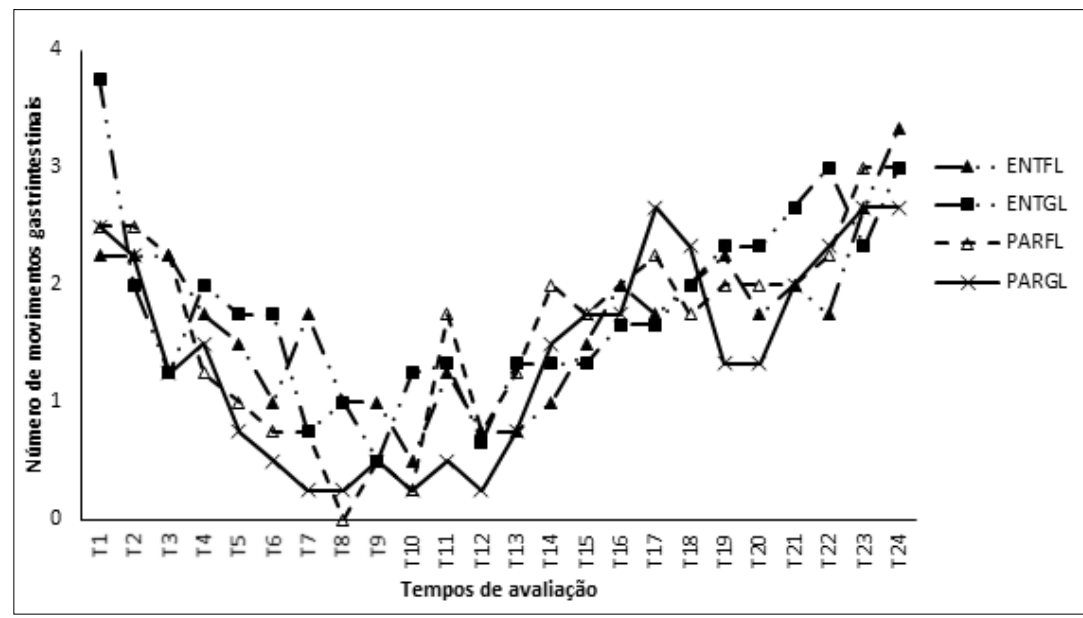

Figura 4: Número de movimentos gastrintestinais propulsivos por minuto no quadrante inferior esquerdo de equinos sob inanição submetidos à fluidoterapia parenteral (PARFL), fluidoterapia enteral associada (ENTGL) ou não a glutamina (ENTFL) e nutrição parenteral total associada à glutamina (PARGL).
Em um estudo avaliando o efeito de diferentes modalidades de reposição hidroeletrolítica (fluidoterapia enteral em fluxo contínuo versus fluidoterapia intravenosa) em equinos desidratados, Dias et al. (2019) não observaram diminuição da motilidade gastrintestinal ao submeterem um grupo de equinos há 36 horas de jejum hídrico e alimentar associadas a duas administrações intravenosas de $1 \mathrm{mg} / \mathrm{kg}$ do diurético furosemida. Estes resultados diferem dos observados no presente estudo, bem como no estudo conduzido por Silveira et al. (2012) que relataram diminuição da motilidade intestinal em equinos advinda de um protocolo de 24 horas de jejum e duas aplicações de furosemida que foi revertida após algumas horas de terapia hidroeletrolítica enteral.

Ribeiro Filho et al. (2012) relataram hipomotilidade intestinal em um protocolo semelhante aos descritos por Silveira et al. (2012) e Dias et al. (2019) e associaram o achado ao desequilíbrio hidro-eletrolítico e ácido base ocasionados pela desidratação. No entanto, no presente estudo, o desenvolvimento de hipomotilidade intestinal na fase de inanição não foi associado há um possível quadro de desidratação, uma vez que foi realizada apenas o protocolo de jejum e os requerimentos diários de manutenção hídrica foram fornecidos em todos os grupos.

Em estudo avaliando os efeitos do jejum em ratos albinos, Obembe et al. (2015) observaram diminuição da motilidade gastrintestinal e concluíram que a ausência de alimento inibia a estimulação vagal 
e, portanto, reduzia a motilidade gastrintestinal. Tais achados corroboram os resultados observados no presente estudo e aqueles relatados por Naylor et al. (2006) ao observarem que períodos prolongados de inanição no equino induz quadro de hipomotilidade gastrintestinal.

Quando considerado o somatório do número de movimentos gastrintestinais propulsivos em todos os quadrantes (Figura 5), não foi identificada interação entre grupo e tempo $(p>0,05)$, e nem diferença $(p>0,05)$ entre o total de movimentos propulsivos na fase 2. Entretanto, foi identificada diferença $(p>0,05)$ entre $o$ total dos grupos na fase 1 e entre o total dos tempos em ambas as fases.

A observação dos valores do somatório permite evidenciar com clareza que a diminuição numérica da quantidade de movimentos gastrintestinais propulsivos ocorreu de forma precoce e brusca nos grupos PARFL e PARGL, concordando com a impressão clínica observada durante o estudo de que o desenvolvimento de quadro de hipomotilidade é precoce nos grupos PARFL e PARGL.

Corroborando com os resultados de Naylor et al. (2006), a realimentação aumentou de forma gradativa o número de movimentos gastrintestinais. A realimentação estimulou um aumento acentuado na motilidade gastrintestinal nas primeiras 48 horas de alimentação. A ativação de reflexo gastrocólico e/ou o aumento da carga calórica podem iniciar essa resposta. Estudos anteriores revelaram que a alimentação ou realimentação após períodos de jejum podem induzir um aumento na frequência, intensidade, e a duração de alguns tipos de atividades mioelétricas intestinais (Ross et al., 1990).

No presente estudo, o somatório do número de movimentos gastrintestinais aumentou a partir do final da inanição até o término da fase de realimentação, retornando a valores similares ao início do estudo, corroborando com os dados de Naylor et al. (2006).

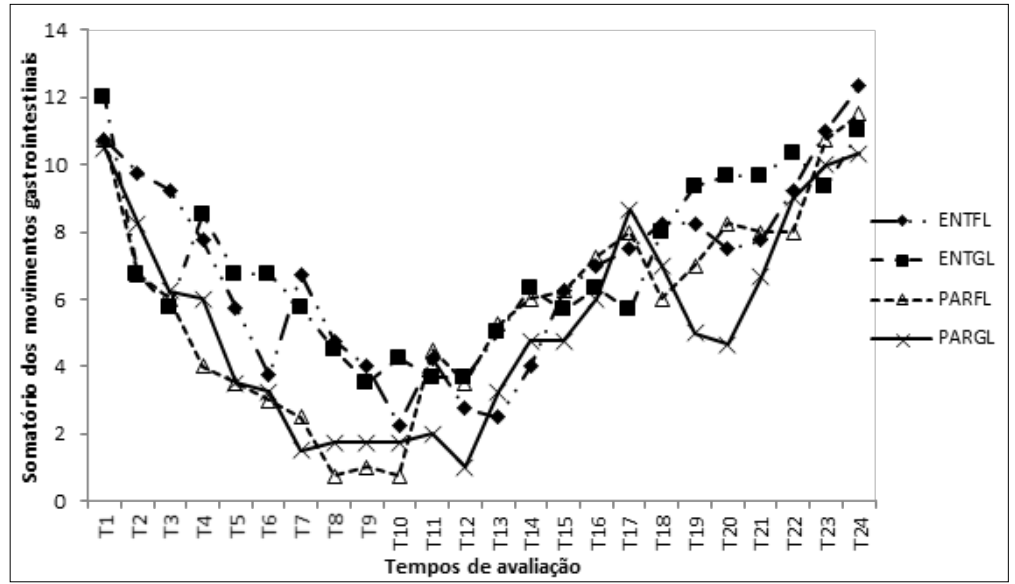

Figura 5: Somatório do número de movimentos gastrintestinais propulsivos por minuto dos quadrantes superior direito, superior esquerdo, inferior direito e inferior esquerdo de equinos sob inanição submetidos à fluidoterapia parenteral (PARFL), fluidoterapia enteral associada (ENTGL) ou não a glutamina (ENTFL) e nutrição parenteral total associada à glutamina (PARGL).

\section{Conclusão}

A motilidade gastrintestinal reduziu drasticamente sob efeito da inanição. Os achados deste estudo alertam para o efeito da inanição sobre a motilidade intestinal predispondo os equinos a síndrome do abdome agudo. Desta forma, restrições alimentares devem ser realizadas, quando necessárias, pelo menor tempo necessário. Pesquisas futuras são necessárias para avaliar as diferentes variáveis influenciando a motilidade gastrintestinal em equinos sob inanição.

\section{Agradecimentos}

Ao CNPq (Conselho Nacional de Desenvolvimento Científico e Tecnológico) pelo auxílio financeiro para a realização deste estudo.

\section{Referências}

BAILEY, P.A.; HAGUE, B.A.; DAVIS, M.; MAJOR, M.D.; ZUBROD, C.J.; BRAKENHOFF, J.E. Incidence of post-anesthetic colic in non-fasted adult equine patients. Canadian Veterinary Journal, v.57, n.12, p.1263-1266, 2016.

CARR, E.A. Enteral/Parenteral Nutrition in Foals and Adult Horses Practical Guidelines for the Practitioner. Veterinary Clinics of North America: Equine Practice, v.34, n.1, p.169-180, 2018.

DOI: 10.1016/j.cveq.2017.11.012
DIAS, D. C. R.; MOREIRA, N. S.; BITTENCOURT, T. C. B. S. C.; OLIVEIRA, D. P.; ERMITA, P. A. N.; SILVA, M. O.; FONSECA, L. A.; RIBEIRO FILHO, J. D. Avaliação comparativa entre diferentes modalidades de reposição hidroeletrolítica em equinos desidratados experimentalmente pela utilização de parâmetros do exame físico e marcadores hematológicos. Revista Brasileira de Ciência Veterinária, v.26, n.2, p.34-41, 2019. DOI: 10.4322/rbcv.2019.007

HOSPES, R.; BLEUL, U. The effect of extended preoperative fasting in mares undergoing surgery of the perineal region. Journal of Equine Veterinary Science, v.27, n.12, p.542-545, 2007. 
LISOWSKI, Z.M.; PIRIE, R.S.; BLIKSLAGER, A.T.; LEFEBVRE, D.; HUME, D.A.; HUDSON, N.P.H. An update on equine postoperative ileus: Definitions, pathophysiology and management. Equine Veterinary Journal, v.50, n.3, p.292-303, 2018. DOI: 10.1111/evj.12801.

LAKANANURAK, N.; GRAMLICH, L. The role of preoperative parenteral nutrition. Nutrients, v.12, n.5, p.1320, 2020.

DOI: 10.3390/nu12051320.

MELO, U.P.; PALHARES, M.S.; GHELLER, V.A.; SILVA FILHO, J.M.; FERREIRA, C.; LEME, F.O.P. Respostas neuroendócrinas à inanição em equinos. Acta Veterinaria Brasilica, v.5, n.1, p.24-32, 2011.

MELO, U.P.; PALHARES, M.S.; OLIVEIRA, J.; FERREIRA, C.; SILVA FILHO, J.M. 2008. Nutrição parenteral em equinos. Arquivos de Ciência Veterinária e Zoologia da Unipar, v.11, n.1, p.63-69, 2008.

MELO, U.P.; PALHARES, M.S.; FERREIRA, C. Íleo adinâmico em equinos: fisiopatologia e tratamento. Arquivos de Ciência Veterinária e Zoologia da Unipar, v.10, n.1, p.49-58, 2007.

NAYLOR, J.M.; POIRIER, K.L.; HAMILTON, D.L.; DOWLING, P.M. The effects of feeding and fasting on gastrointestinal sounds in adult horses. Journal of Veterinary Internal Medicine, v.20, n.6, p.408-1413, 2006. DOI: 10.1111/j.1939-1676.2006.tb00759.x.

OBEMBE, A.O.; OKON, V.E.; OFUTET, E.O.; AYITU, R.A. Effect of fasting on intestinal motility and transit in Albino Wistar rats. Trends in Medical Research, v.10, n.3, p.63-68, 2015. DOI: 10.3923/tmr.2015.63.68.

PUCCI, N.D.; FONTES, B.; POGGETTI, R.S. Avaliação de um esquema de realimentação utilizado após 43 dias de jejum voluntário. Revista de Nutrição, v.21, n.5, p.503-512, 2008. DOI: 10.1590/S1415-52732008000500003.

RIBEIRO FILHO, J.D.; ALVES, G.E.S.; DANTAS, W.M.F. Tratamentos da compactação experimental do cólon maior de equinos com hidratação enteral, intravenosa e sene (Cassia augustifolia Vahl). Revista Ceres, v.59, n.1, p. 407-410, 2012.

ROSS, M.W.; CULLEN, K.K.; RUTKOWSKI, J.A. Myoelectric activity of the ileum, cecum, and right ventral colon in ponies during interdigestive, nonfeeding, and digestive periods. American Journal Veterinary Research, v.51, n.4, p.561-566, 1990.
SALCICCIA, A.; GOUGNARD, A.; GRULKE, S.; DE LA REBIĖRE DE POUYADE, G.; LIBERTIAUX, V.; BUSONI, V.; SANDERSEN, C.; SERTEYN, D. Gastrointestinal effects of general anaesthesia in horses undergoing non abdominal surgery: focus on the clinical parameters and ultrasonographic images. Research in Veterinary Science, v. 124, p.123-128, 2019. DOI: 10.1016/j. rvsc.2019.03.011.

SEAHORN, J.L.; SEAHORN, T.L. Fluid therapy in horses with gastrointestinal disease. Veterinary Clinics of North America: Equine Practice, v.19, n.3, p.665-679, 2003.

SPEIRS, V.C. Exame Clínico de Equinos. 1.ed. Porto Alegre: Editora Artmed, 1999. 366p.

SILVEIRA, C. O.; RIBEIRO FILHO, J. D.; DA CRUZ, A. L.; MENESES, R. M.; DANTAS, W. D. M. F.; GUIMARÃES, J. D. Avaliação de equinos submetidos à hidratação enteral com soluções eletrolíticas isotônicas contendo diferentes fontes de energia e cálcio. Archives of Veterinary Science, v.17, n.1, p.2231, 2012. DOI: http://dx.doi.org/10.5380/avs.v17i1.22640

TAPIO, H.; RAEKALLIO, M.R.; MYKKÄNEN, A.; MÄNNIKKÖ, S.; SCHEININ, M.; BENNETT, R.C.; VAINIO, O. Effects of vatinoxan on cardiorespiratory function and gastrointestinal motility during constant-rate medetomidine infusion in standing horses. Equine Veterinary Journal, v.51, n.5, p.646-652. 2019. DOI: 10.1111/evj.13085.

VANDERBROEK, A.R.; REEF, V.B.; AITKEN, M.R.; STEFANOVSKI, D.; SOUTHWOOD, L.L. Assessing gastrointestinal motility in healthy horses comparing auscultation, ultrasonography and an acoustic gastrointestinal surveillance biosensor: a randomised, blinded, controlled crossover proof of principle study. Equine Veterinary Journal, v.51, n.2, p.246-251, 2019. DOI: $10.1111 /$ evj.12990.

WILLIAMS, S.; HORNER, J.; ORTON, E.; GREEN, M.; MCMULLEN, S.; MOBASHERI, A.; FREEMAN, S.L. Water intake, faecal output and intestinal motility in horses moved from pasture to a stabled management regime with controlled exercise. Equine Veterinary Journal, v.47, n.1, p.96-100, 2015. DOI: 10.1111/evj.12238 